\title{
Higienização bucal em pacientes com deformidades nas mãos: uso de adaptador para escova dental
}

\section{Oral hygiene in patients with deformity hands: adapter for use dental brush}

\author{
Cristina Maria da Paz Quaggio* \\ Maira Giampietro de Almeida Nogueira** \\ Claudia de Almeida Prado Piccino Sgavioli** \\ Graziela de Almeida Prado Piccino Marafiotti ${ }^{* * *+}$ \\ Solange de Oliveira Braga Franzolin ${ }^{* * * * *}$ \\ Marcos da Cunha Lopes Virmond ${ }^{* * * * * * *}$
}

\section{Resumo}

Objetivo: avaliar o efeito do uso de uma adaptação para escova dental em indivíduos afetados pela hanseníase com garra e avaliar sua contribuição na higiene bucal. Sujeitos e método: estudo realizado no Instituto Lauro de Souza Lima, com amostra de dez pacientes com idade acima de 18 anos, apresentando garra ulnar, ou garra ulnar mediano no membro dominante, entre 2013 e 2016. Foram realizadas a identificação dos pacientes, de seu perfil social e da forma clínica da hanseníase e a análise da higienização bucal pelo Índice de Higiene Oral Simplificado (IHO-S). Cada componente desse índice é avaliado em uma escala de 0 a 3. O resultado do IHO-S é dado pela média dos pontos de cada dente, assim classificados: 0 a 1 - higiene satisfatória; 1,1 a 2 - higiene regular; 2,1 a 3 - higiene deficiente. Resultados: o IHO-S dos pacientes foi medido em três momentos: antes da entrega do adaptador; uma semana de uso contínuo do adaptador, 15 dias de uso contínuo do adaptador. Observaram-se valores menores da média e mediana do IHO-S com o uso do adaptador, de acordo com o tempo. O teste estatístico ANOVA confirmou a diferença significativa entre os grupos ( $p=0,002196)$. $O$ teste Tukey registrou a diferença do $1^{\circ}$ momento em relação ao $2^{\circ}(p=0,02509)$ e ao $3^{\circ}(p=0,002199)$; não houve diferença estatística significativa entre o $2^{\circ}$ e o $3^{\circ}$ momentos. O teste de Kruskal-Wallis confirmou a diferença significativa entre os grupos ( $p=0,005887$ ). Conclusões: a tecnologia assistiva pode gerar melhora na capacidade funcional. Mesmo considerando o pequeno universo deste estudo, conclui-se que as adaptações podem contribuir para a melhora da qualidade de vida e também para a melhor qualidade da higiene bucal de tal população.

Palavras-chave: Hanseníase. Higiene bucal. Tecnologia assistiva.

\section{Introdução}

A hanseníase é uma doença que se caracteriza por comprometimento da pele e de nervos periféricos, podendo causar alteração da sensibilidade e até incapacidade motora. É uma doença causada pelo Mycobacterium Leprae e sua transmissão ocorre pelo contato direto pelas vias aéreas superiores com pacientes infectados pelo bacilo ${ }^{1}$.

Normalmente, o comprometimento no membro superior mais frequente é o do nervo ulnar, na região do cotovelo, podendo haver a combinação de lesão do nervo ulnar e mediano, resultando na perda da sensibilidade e paralisia da musculatura intrínseca da mão ${ }^{2}$.

Doutoranda em Biologia Oral na Universidade do Sagrado Coração. Docente da Universidade do Sagrado Coração. Terapeuta Ocupacional no Instituto Lauro de Souza Lima, Bauru, São Paulo, Brasil.

Dentista do Instituto Lauro de Souza Lima.

Doutor em Dentística pela Faculdade de Odontologia de Bauru da Universidade de São Paulo. Diretora Geral da Diretoria Executiva da Fundação Regional de Saúde do Município de Bauru, Bauru, São Paulo, Brasil.

Doutora em Ciências da Saúde, Fisiopatologia e Clínica Médica, UNESP-FMB.

Doutor em Fisiopatologia em Clínica Médica pela Faculdade de Medicina Botucatu, Unesp. Docente da Universidade Paulista e professor assistente da Universidade do Sagrado Coração.

****** Pesquisador científico VI do ILSL/CCD - SES-SP. Professor orientador do Programa de Pós-Graduação em Biologia Oral da Universidade do Sagrado Coração, Bauru, São Paulo, Brasil. 
A paralisia da musculatura intrínseca da mão pode levar a cinco principais alterações motoras: perda da habilidade de abdução e adução dos dedos, o que dificulta atividades que envolvam o separar dos dedos e pegar objetos esféricos grandes, digitar, tocar piano, aparar água na palma das mãos, dificuldade na flexão das metacarpofalangianas, que se trata da consequência mais séria da paralisia dos músculos intrínsecos, ocasionando a presença de garra, dificuldade na extensão das interfalangianas distais, que impede uma preensão adequada, provocando áreas de pressão excessiva nas pontas dos dedos, dificuldade na flexão e oposição do $5^{\text {o dedo, }}$ que gera a inversão do arco metacarpiano transverso, criando uma mão palmar, sem arco e perda da oposição do polegar ${ }^{2}$.

Essas deformidades, certamente, comprometem a realização das atividades de vestuário, alimentação e higiene, dentre elas, a higiene bucal. Atualmente, os profissionais da saúde têm se preocupado em tratar seus pacientes de forma multidisciplinar, pois a boa saúde sistêmica do indivíduo depende de muitos fatores, dentre eles, a saúde bucal. Estudos mostram que o risco de endocardite bacteriana, descontrole da glicemia e problemas renais pode estar relacionado à falta de manutenção da saúde bucal ${ }^{3}$.

A condição da saúde bucal pode interferir na qualidade de vida do indivíduo. A cárie dental e a doença periodontal são as doenças mais prevalentes na população brasileira, fato que as coloca como as maiores causas de perda de elementos dentários. No que se refere à qualidade de vida, não se leva em consideração apenas a saúde física, mas também o contexto social em que o indivíduo se insere e as implicações para sua vida ${ }^{4}$. Cada indivíduo tem necessidades e riscos específicos, que se traduzem em um perfil próprio de saúde-doença.

Alguns indivíduos não têm boa saúde bucal por apresentarem dificuldades para a realização da higiene bucal, como os indivíduos afetados pela hanseníase, pelo fato de perderem a sensibilidade protetora da mão e pelas alterações motoras advindas do dano neural nessa doença. O prejuízo que a hanseníase traz para a saúde bucal mostrou-se correlacionado à dificuldade de realizar a higiene bucal, sendo que diversos motivos podem dificultar a higiene, como os episódios reacionais em que o paciente fica com sua saúde sistêmica comprometida, tornando mais difícil a execução dos hábitos de higiene corporal, incluindo também a bucal ${ }^{5,6}$.

As deformidades decorrentes da hanseníase podem alterar a função adequada nas atividades de vida diária (AVDs), como é o caso da escovação dental para a higiene bucal, sendo necessário o uso de uma adaptação, considerada uma forma de ajuda técnica ao deficiente, avaliada como dispositivo de assistência ${ }^{7}$. Conforme o conceito adotado pelo Secretariado Nacional para a Reabilitação e Integração das Pessoas com Deficiência de Portugal (SNRIPD), no Catálogo Nacional de Ajudas Técnicas
(CNAT) $^{8}$, a tecnologia assistiva, também chamada de ajudas técnicas, pode ser considerado todo o processo que visa ao contexto do produto propriamente dito até sua confecção, visando à prevenção, além de minimizar ou neutralizar a deficiência e permitir a melhora da autonomia e da qualidade de vida dos indivíduos ${ }^{8}$.

No entanto, tecnologia assistiva são todas e quaisquer ferramentas, recursos ou estratégias e processos desenvolvidos e utilizados com a finalidade de possibilitar a independência e a autonomia à pessoa com deficiência. São considerados como tecnologia assistiva desde artefatos simples, como uma colher adaptada ou um lápis com uma empunhadura mais grossa para facilitar a preensão, até sofisticados programas especiais de computador visando à acessibilidade ${ }^{9}$.

Estudos demonstram que as adaptações promovem e facilitam a independência funcional, caracterizando, assim, a melhora do padrão preensor, impedindo ou prevenindo a gravidade das deformidades $^{10,11}$. Devido ao comprometimento motor e sensitivo na hanseníase, dificilmente se pode dispensar o uso de adaptações na rotina do dia a dia do indivíduo.

O cirurgião-dentista tem o compromisso e a responsabilidade de passar a instrução de higiene oral ao paciente, demostrando a técnica adequada para cada indivíduo ${ }^{12}$. Possibilitar a melhoria da qualidade de saúde bucal do indivíduo implica a melhora de sua saúde e pode contribuir para a melhora da qualidade de vida.

Assim, este estudo teve como objetivo caracterizar uma amostra de pacientes com hanseníase e apresentar o uso de um adaptador (tecnologia assistiva) na higienização oral, com vistas a promover a sua saúde bucal e avaliar sua eficiência utilizando um índice de higiene oral específico.

\section{Sujeitos e método}

O estudo foi submetido à avaliação pelo Comitê de Ética em Pesquisa, sob protocolo no ${ }^{-069 / 12}$, da Universidade Sagrado Coração e à apreciação do Comitê de Ética do Instituto Lauro de Souza Lima, Bauru, São Paulo. Os interessados em participar receberam um termo de consentimento livre e esclarecido para assinar e autorizar sua participação na pesquisa, que teve início em outubro de 2013 e término em abril de 2016. O termo foi elaborado de acordo com a Resolução no $196 / 1996$, sobre pesquisas envolvendo seres humanos.

Participaram deste estudo dez indivíduos afetados pela hanseníase, já tratados ou em tratamento com poliquimioterapia, com garra ulnar e/ou garra ulnar mediano do membro superior dominante, que apresentavam necessidade de acompanhamento dentário, com idade igual ou superior a 18 anos, que estavam internados na enfermaria ou em atendi- 
mento no ambulatório dos setores de Odontologia e de Terapia Ocupacional do Instituto Lauro de Souza Lima, em Bauru, SP. Os critérios de exclusão compreenderam pessoas afetadas pela hanseníase com idade inferior a 18 anos, pacientes sem deformidade aparente do membro dominante, baixa acuidade visual e auditiva e com prótese dentária total.

Para a coleta de dados, foram utilizados: anamnese: identificação e perfil social do paciente: nome, sexo, data de nascimento, escolaridade, forma clínica da hanseníase e informações sobre a saúde geral e o Índice de Higiene Oral Simplificado (IHO-S): para o registro da condição de higiene bucal do paciente, por meio do exame clínico de superfície de alguns dentes pré-estabelecidos pelo índice ${ }^{13}$.

Para a avaliação da higiene bucal, foi utilizado o instrumento IHO-S ${ }^{13}$, que mede a área de superfície do dente coberta por resíduo (placa) e cálculo; sendo examinadas as faces vestibulares dos dentes: 16 (Vestibular do $1^{\circ}$ molar superior direito), 11 (Vestibular do incisivo central superior direito), 26 (Vestibular do $1^{\mathrm{o}}$ molar superior esquerdo), 36 (Lingual do $1^{\circ}$ molar inferior esquerdo), 31 (Vestibular do incisivo central inferior esquerdo) e 46 (Lingual do $1^{\circ}$ molar inferior direito). Cada componente desse índice (resíduo e cálculo) é avaliado em uma escala de 0 a 3, baseado na fração da superfície dental coberta por placa e cálculo (Figura 1).

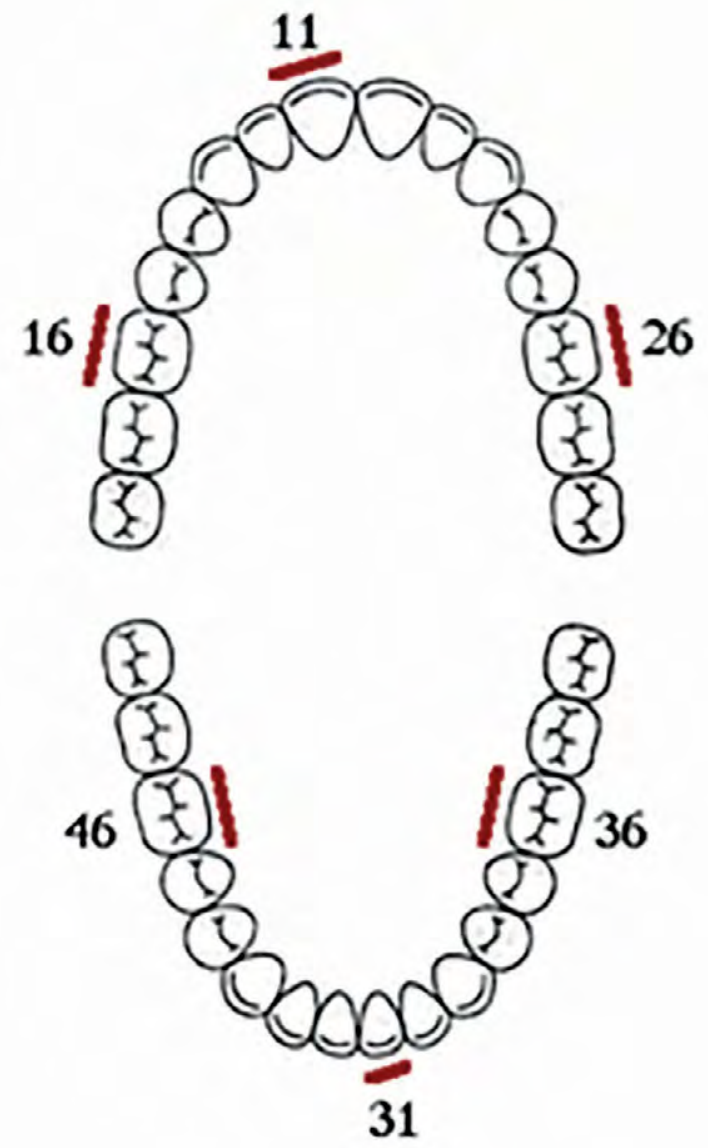

Figura 1 - Esquema dos dentes e das faces avaliadas no IHO-S Fonte: Salud Dental Para Todos ${ }^{14}$.
Inicialmente, o paciente foi avaliado em consultório odontológico pelo cirurgião-dentista, que registrou o seu IHO-S; em seguida, instruiu-o sobre a higiene bucal, oferecendo um kit com duas escovas de dente e um creme dental. Nesse momento, o terapeuta ocupacional entregou o adaptador para escova dental, sugerindo que a usasse dentro da sua rotina doméstica própria de higienização oral. Após uma semana, a segunda avaliação foi feita com o novo registro de IHO-S, o que foi repetido depois de quinze dias de uso do adaptador (do dia da entrega). Todas as avaliações odontológicas foram realizadas de maneira individual, pelo mesmo cirurgião-dentista, usando equipamento de proteção individual recomendado, no mesmo consultório, sob luz artificial, utilizando espelho bucal plano número 5 e espátulas de madeira. O resultado do IHO-S é dado pela média dos pontos de cada dente, assim classificados: 0 a 1 - higiene satisfatória; 1,1 a 2 - higiene regular; 2,1 a 3 - higiene deficiente.

Os resultados foram apresentados por meio da frequência absoluta, média e mediana. Para comparar os três momentos, foram aplicados os testes ANOVA, Tukey e Kruskal-Wallis, utilizando o programa computacional PAST versão 1.74, adotando-se 95\% de nível de confiança.

\section{Resultados}

Os resultados estão relacionados com a caracterização da amostra estudada. Os dez pacientes tinham idade entre 22 e 60 anos, sendo que oito eram do sexo masculino. Em relação à procedência, observou-se que seis eram do estado de São Paulo e os demais eram do Distrito Federal e dos estados de Minas Gerais, Rondônia, Rio Grande do Sul e Tocantins. Quanto à escolaridade, quatro apresentavam ensino médio completo, três com ensino fundamental incompleto e os outros três tinham ensino fundamental completo, ensino médio incompleto e superior completo. No item estado civil, seis declararam-se solteiros, três casados e um com união consensual.

Quanto às outras variáveis pesquisadas, os resultados mais frequentes foram: oito pacientes usavam medicamento, seis não eram fumantes, oito não consumiam álcool e, na variável atividade social, seis frequentavam reuniões sociais (forró, bailes, etc.) e três iam à igreja.

Em relação à forma clínica, seis pacientes apresentavam a forma dimorfa, quatro a forma virchoviana, seis eram pacientes com garra ulnar e quatro com garra ulnar mediano no membro dominante.

Quanto às dificuldades em realizar as atividades do dia a dia, seis pacientes relataram que, às vezes, têm dificuldades na realização de alguma atividade.

A Tabela 1 apresenta o resultado do IHO-S dos pacientes nos três momentos: antes da entrega do 
adaptador, uma semana após uso contínuo do adaptador e quinze dias após uso contínuo do adaptador. Observaram-se valores menores da média e mediana do IHO-S com o uso do adaptador, de acordo com o tempo.

O teste estatístico ANOVA confirmou a diferença significativa entre os grupos (valor de $p=$ $0,002196)$. O teste Tukey registrou a diferença do $1^{\circ}$ momento em relação ao $2^{\mathrm{o}}$ (valor de $\mathrm{p}=0,02509$ ) e do $1^{\circ}$ em relação ao $3^{\circ}$ (valor de $p=0,002199$ ); não houve diferença estatística significativa entre o $2^{\circ}$ e o $3^{\text {o }}$ momentos. O teste estatístico Kruskal-Wallis confirmou a diferença significativa entre os grupos (valor de $\mathrm{p}=0,005887$ ).

Tabela 1 - IHO-S dos pacientes avaliados nos três momentos

\begin{tabular}{l|r|r|r}
\hline Paciente & $\begin{array}{c}\text { Antes da } \\
\text { adaptação }\end{array}$ & $\begin{array}{c}\text { 7 dias após } \\
\text { a adaptação }\end{array}$ & $\begin{array}{c}\text { 15 dias após a } \\
\text { adaptação }\end{array}$ \\
\hline 1 & 1,33 & 1,5 & 0,66 \\
2 & 1,66 & 0,83 & 0,15 \\
3 & 0,83 & 0,66 & 0,50 \\
4 & 0,66 & 1,0 & 0,66 \\
5 & 1,0 & 0,66 & 0,5 \\
6 & 0,33 & 0,16 & 0,33 \\
7 & 1,66 & 0,66 & 0,44 \\
8 & 1,5 & 0,5 & 0,5 \\
9 & 2,5 & 1,16 & 0,66 \\
10 & 2,5 & 0,66 & 1,16 \\
Média & 1,40 & 0,78 & 0,56 \\
Mediana & 1,42 & 0,66 & 0,5 \\
\hline N:10 & &
\end{tabular}

Fonte: elaboração dos autores.

Em relação às deformidades de membro superior, seis avaliados apresentavam garra ulnar e outros quatro, garra ulnar mediano, o que contribuiu para a dificuldade no ato de pegas a escova. Essa característica, além da deformidade, está associada também com a fraqueza da musculatura intrínseca da mão, que ocorre nos dois casos da lesão, levando a déficits funcionais consideráveis (Figuras 2 e 3).

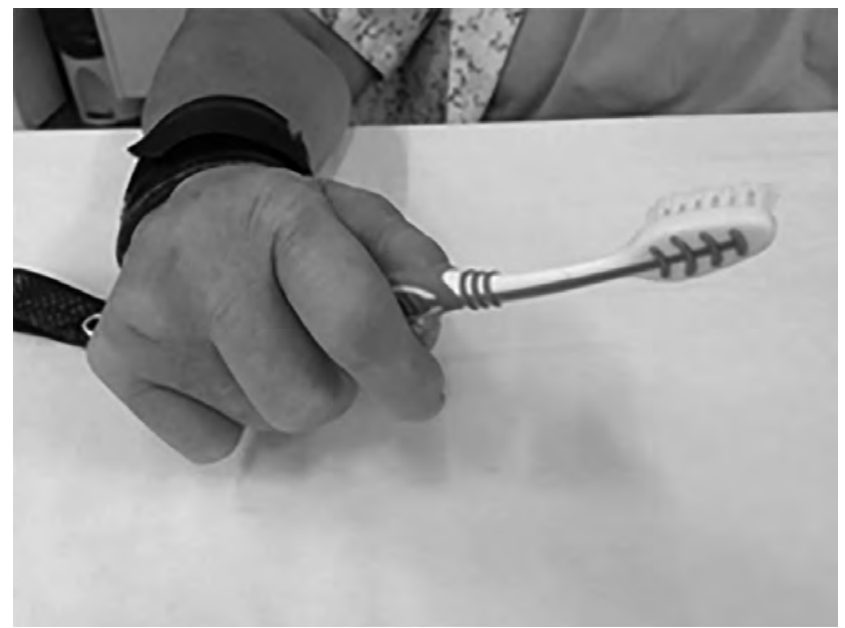

Figura 2 - Empunhadura de escova sem adaptador

Fonte: elaboração dos autores.

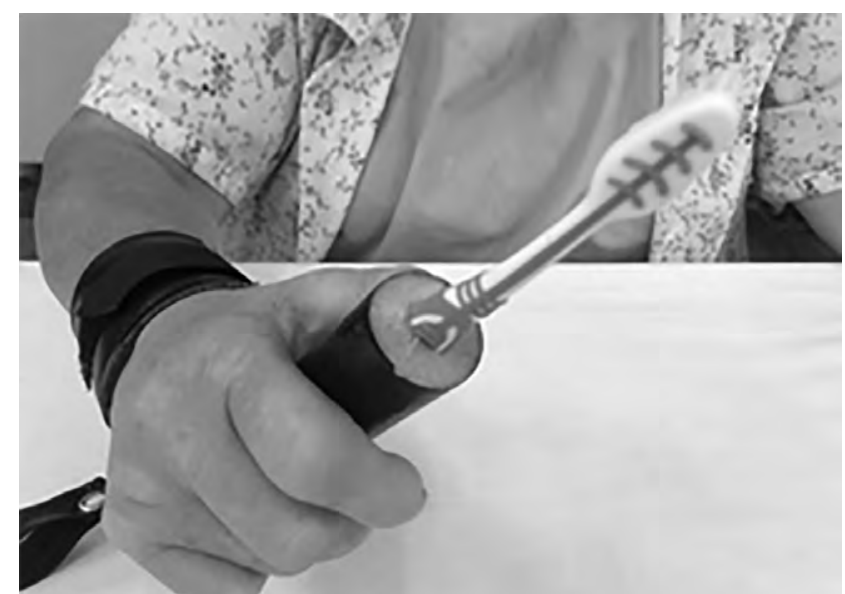

Figura 3 - Empunhadura de escova com adaptador

Fonte: elaboração dos autores.

\section{Discussão}

$\mathrm{Na}$ amostra estudada, a idade dos pacientes variou entre 22 e 60 anos, sendo a média de idade de 34 anos. Quanto à escolaridade, quatro apresentavam o ensino médio completo e os demais se caracterizavam por ensino fundamental incompleto, sendo que apenas um participante apresentou nível universitário. Tal informação é pertinente, uma vez que um estudo realizado ${ }^{5}$ constatou que o comprometimento e o interesse em realizar avaliação bucal foram constatados nos indivíduos que apresentaram um maior grau de escolaridade.

Muitos fatores contribuem para o entendimento que a pessoa tem em relação ao seu estado de saúde, entendido como autopercepção, e considera-se que fatores como idade, aspectos demográficos, sexo, raça, escolaridade e até mesmo a predisposição contribuem para o acesso à informação sobre os cuidados preventivos e consequentes autopercepção da saúde bucal ${ }^{15,16}$.

Pessoas com deficiências físicas têm uma maior dificuldade em realizar higienização oral, particularmente aquelas com alterações em membros superiores. De fato, um estudo ${ }^{17}$ que utilizou o mesmo instrumento de avaliação em indivíduos em idade escolar de 14 a 35 anos, com deficiência física e mental, verificou que aqueles que apresentavam deficiência física também revelaram índice de higiene oral deficiente.

Na hanseníase, os nervos mais comprometidos no membro superior são o ulnar, mediano e radial, ocorrendo primeiramente a lesão do nervo ulnar, seguida da lesão do mediano, e a lesão do ulnar mediano é considerada a mais grave e completa devido ao comprometimento da região tenar, hipotenar e à perda de oponência do polegar. É certo que essas deformidades, por sua vez, ocorrem em fase ativa do indivíduo, possibilitando o afastamento de suas atividades profissionais e influenciando sua inserção social. 
Considerando-se as formas clínicas da doença, a amostra dividiu-se entre casos dimorfos e virchovianos. Na hanseníase, a forma virchoviana tem maior possibilidade de apresentar deformidades físicas significativas, o que confirma também nossa amostra. Isso se explica pelo processo longo da doença e pelos estados reacionais frequentes que acontecem nessa patologia ${ }^{18}$. Mais que isso, a doença periodontal pode ser um fator desencadeador dessas reações ${ }^{19}$. De fato, os mediadores proinflamatórios envolvidos nos processos imunopatológicos para a infecção gengival e para as reações hansênicas são idênticos ${ }^{20}$. Assim, o monitoramento efetivo do tratamento dessas infecções poderia diminuir a incidência do dano neural e das incapacidades nos casos de hanseníase, assim como a perda dental de modo geral.

Outro fator está envolvido nessa equação. Um estudo realizado sobre a saúde bucal das pessoas afetadas pela hanseníase ${ }^{5}$ refere que a dificuldade com a higiene bucal também está ligada ao diagnóstico tardio da doença, com a consequente sequela neuromotora, dificultando a realização da higiene oral. Tal estudo observou, também, que $27,80 \%$ dos pacientes relataram que, na hanseníase, os dentes ficam frágeis e que 5,55\% afirmaram ter dificuldades em segurar a escova ${ }^{5}$.

No presente estudo, os participantes apresentaram uma diferença significativa em seu índice de placa após o uso do adaptador (valor de $p=0,02509$ ), indicando que o uso da adaptação pode favorecer a escovação e, muito provavelmente, diminuir a ocorrência de doença periodontal. De fato, a dificuldade motora e articular em realizar a tarefa de higienização bucal pode ser severamente comprometida nesses casos, tanto pela própria dificuldade motora como pelo desinteresse consequente da dificuldade. $\mathrm{O}$ uso de um adaptador, que facilita a atividade, reduz esses dois componentes. Preliminarmente, o uso do equipamento apresentado nesta pesquisa parece indicar essa possibilidade.

\section{Conclusão}

Devido às deformidades ocasionadas pela doença, as pessoas afetadas pela hanseníase apresentam dificuldades nas atividades de vida diária. Adaptações simples, como as incluídas na tecnologia assistiva, podem facilitar e complementar essa diminuição de funcionalidade, conforme demonstrado neste estudo.

Mesmo com o pequeno universo do estudo, pode-se concluir que, para a questão da higiene bucal, a adaptação proposta revelou resultados positivos para o Índice de Higiene Oral Simplificado, o que, continuadamente, poderia resultar em melhora da saúde bucal dos indivíduos. Com os avanços tecnológicos, seria oportuno realizar uma pesquisa comparativa entre esse método adaptado à escova com uso de escovas elétricas.

\section{Agradecimento}

Agradecemos à Fundação Paulista contra Hanseníase, que financiou esta pesquisa.

\section{Abstract}

Objective: to evaluate the effect of using a toothbrush adaptation on individuals affected by leprosy with claw-hand and to evaluate its contribution for oral hygiene. Subjects and method: a study carried out at the Lauro de Souza Lima Institute between 2013 and 2016 with a sample of ten patients over 18 years of age presenting ulnar claw or ulnar/median claw in the dominant limb. Methods included the identification and social profiling of the patient, clinical form of leprosy, and analysis of oral hygiene by the Simplified Oral Hygiene Index $(\mathrm{OHI}-\mathrm{S})$. Each component of this index is evaluated on a scale from 0 to 3. The OHI-S result is determined by the mean score of each tooth, thus classified as 0 to 1 satisfactory hygiene, 1.1 to 2 - regular hygiene, and 2.1 to 3 - poor hygiene. Results: the OHI-S of patients was measured at the following three moments: before handing out the adapter, after one week of continuous use of the adapter, and after 15 days of continuous use of the adapter. There were lower mean and median $\mathrm{OHI}$ $-S$ values with the use of the adapter according to the period. The ANOVA statistical test confirmed the significant difference between the groups ( $p=0.002196$ ). Tukey's test recorded the difference of the $1^{\text {st }}$ moment relative to the $2^{\text {nd }}(p=0.02509)$ and $3^{\text {rd }}(p=0.002199)$ moments. There was no significant statistical difference between $2^{\text {nd }}$ and $3^{\text {rd }}$ moments. The Kruskal-Wallis test confirmed the significant difference between the groups $(p=0.005887)$. Conclusions: assistive technology may improve functional capacity. Even when considering the small universe of this study, it may be concluded that the adaptations can contribute to the improvement of quality of life and to better quality of oral hygiene of this population.

Keywords: Leprosy. Oral hygiene. Assistive technology.

\section{Referências}

1. Brasil. Ministério da Saúde. Guia para o controle da hanseníase. Brasília: Ministério da Saúde; 2002.

2. Lehman LF, Orsini MBP, Grossi MAF, Villarroel MF. A mão na hanseníase. In: Freitas PP. Reabilitação da mão. São Paulo: Atheneu; 2005. p. 301-18.

3. Wilson W, Taubert KA, Gewitz M, Lockhart PB, Baddour LM, Levison M, et al. Prevention of infective endocarditis: guidelines from the American Heart Association. J Am Dent Assoc 2008; 139Suppl:3S-24S.

4. Silva SRC, Valseki Junior A. Avaliação da condição de saúde bucal dos idosos em um município brasileiro. Rev Panam Salud Publica [on-line] 2000 Out [acesso em 25 ago. 2016]; 8(4):268-71. Disponível em URL: http://www.scielosp.org/pdf/ $\mathrm{rpsp} / \mathrm{v} 8 \mathrm{n} 4 / 3553$.pdf.

5. Almeida JRS, Alencar CH, Barbosa JC, Dias AA, Almeida MEL. Autopercepção de pessoas acometidas pela hanseníase sobre sua saúde bucal e necessidade de tratamento. Ciênc Saúde Colet 2013; 8(3):817-26. 
6. Costa A, Nery J, Oliveira M, Cuzzi T, Silva M. Oral lesions in leprosy. Indian J Dermatol Venereol Leprol 2003; 69(6):3815. Pubmed PMID:17642946.

7. Cavalcante A, Galvão CA. Adaptação ambiental e doméstica. In: Cavalcante A, Galvão CA. Terapia ocupacional: fundamentação e prática. Rio de Janeiro: Guanabara Koogan; 2007. p. 420-6.

8. Kastrup V. Novas tecnologias cognitivas: o obstáculo e a invenção. In: Pellanda NMC, Pellanda EC, organizadores. Ciberespaço: um hipertexto com Pierre Lévy. Porto Alegre: Artes e Ofícios; 2000.

9. Hazard D, Galvão Filho TA, Rezende ALA. Inclusão digital e social de pessoas com deficiência: textos de referência para monitores de telecentros. Brasília: UNESCO; 2007. 73 p.

10. Marciano L, Baccarelli R. Prevenção de incapacidades e reabilitação em hanseníase. Bauru: ILSL; 2003.

11. Aides techniques et ergotherapie: position officielle del'Association canadienne des ergotherapeutes. Can J Occup Ther 2003; 70(2):116-8.

12. Candelária LF, Teramoto L, Lopes AMS, Ortiz G, Moraes AT. Estudo sobre motivação e reforço de motivação em escovação dentária, em escolares de 7 a 10 anos. Rev Odontol Unesp [on-line]. 1989 [acesso em 25 ago. 2016]; 18:217-23. Disponível em URL: http://www.revodontolunesp.com.br/files/ v18n\%C3\%9Anico/v18nua21.pdf.

13. Greene JC, Vermillion JR. Simplified oral hygiene index. J Am Dent Assoc 1964; 68(1):7-13.

14. Salud Dental Para Todos. ISHO: índice simplificado de higiene oral [on-line]. Buenos Aires: 2014 [acesso em 25 abr. 2016]. Disponível em URL: http://www.sdpt.net/ID/indicesimplificadohigieneoral.htm.

15. Mathias RE, Atchison KA, Lubben JE, Jong FD, Schweitzer SO. Factors affecting self-ratings of oral health. J Public Health Dent 1995; 55(4):197-204.

16. Martins AMEBL, Barreto SM, Pordeus IA. Autoavaliação de saúde bucal em idosos: análise com base em modelo multidimensional. Cad Saúde Pública 2009 25(2):421-35.

17. Queiroz FS, Rodrigues MMLF, Cordeiro Junior GA, Oliveira AB, Oliveira JD, Almeida ER. Avaliação das condições de saúde bucal de portadores de necessidades especiais. Rev Odontologia Unesp 2014; 43(6):396-401.

18. Ramadan W, Mourad B, Fadel W, Ghoraba E. Clinical, electrophysiological, and immunopathological study of peripheral nerves in Hansen's disease. Lepr Rev 2001 72(1):35-49.

19. Motta AC, Furini RB, Simão JC, Vieira MB, Ferreira MA, Komesu MC, et al. Could leprosy reaction episodes be exacerbated by oral infections? Rev Soc Bras Med Trop 2011; 44(5):633-5.

20. Cortela DCB, Souza Junior AL, Virmond MCL, Ignotti E. Inflammatory mediators of leprosy reactional episodes and dental infections: a systematic review. Mediators of Inflammation 2015; Article ID 548540: 15.

\section{Endereço para correspondência:}

Cristina Maria da Paz Quaggio

Rua Julio Rodrigues Horta 1-165 Jardim Estoril III

17016360 Bauru, SP

Fone: (14) 997136331

E-mail: cristina.quaggio@gmail.com

Recebido: 21/09/2016. Aceito: 11/01/2017. 